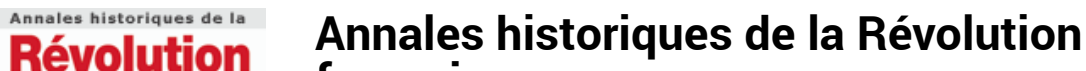

française française

344 | avril-juin 2006

La prise de parole publique des femmes

\section{Pauline Léon, une républicaine révolutionnaire}

\section{Claude Guillon}

\section{(2) OpenEdition}

\section{Journals}

\section{Édition électronique}

URL : https://journals.openedition.org/ahrf/6213

DOI : 10.4000/ahrf.6213

ISSN : 1952-403X

Éditeur :

Armand Colin, Société des études robespierristes

Édition imprimée

Date de publication : 1 juin 2006

Pagination : 147-159

ISSN : 0003-4436

\section{Référence électronique}

Claude Guillon, «Pauline Léon, une républicaine révolutionnaire », Annales historiques de la Révolution française [En ligne], 344 | avril-juin 2006, mis en ligne le 01 juin 2009, consulté le 22 avril 2022. URL : http://journals.openedition.org/ahrf/6213 ; DOI : https://doi.org/10.4000/ahrf.6213

Ce document a été généré automatiquement le 22 avril 2022.

Tous droits réservés 


\title{
Pauline Léon, une républicaine révolutionnaire
}

\author{
Claude Guillon
}

1 Pauline Léon a peu retenu l'attention des historiens, qu'ils lui préfèrent Claire Lacombe, actrice réputée pour sa beauté ou qu'ils jugent les Citoyennes républicaines révolutionnaires manipulées par des hommes comme Théophile Leclerc, que Pauline épousera ${ }^{1}$. Il faut attendre les années 1960 pour que Robert Barrie Rose consacre à Pauline Léon une notice biographique dans The Enragés, Socialists of the French Revolution? (1965) à égalité avec Jean-François Varlet ou Claire Lacombe, et que Marie Cerati publie son Club des citoyennes républicaines révolutionnaires (1966). Dominique Godineau contribue, avec Citoyennes tricoteuses (1988), à l'appréciation de leur action sans rien apporter de nouveau sur Pauline. Enfin, j'ai publié en 1993 une biographie de Théophile Leclerc et Pauline Léon ${ }^{2}$, ici revue et complétée, notamment pour la période postérieure à 1804 .

2 Jeune, célibataire sans enfant, fille d'artisans, Pauline Léon est représentative des femmes de la sans-culotterie parisienne qui s'identifient, dès ses premiers jours, à la Révolution: «J'éprouvais le plus vif enthousiasme et quoique femme je ne demeurai pas oisive; l'on me vit du matin au soir animer les citoyens contre les artisans de la tyrannie [...], barricader les rues et exciter les lâches à sortir de leurs maisons ${ }^{3}$ ». Agissant partout où elle le peut, des émeutes aux sociétés populaires qui acceptent les femmes, elle est de celles qui vont tenter de faire reconnaître la place des militantes non seulement dans l'action révolutionnaire mais dans le nouvel ordre social.

Née à Paris le 28 septembre 1768, Pauline Léon a 21 ans en 1789. Son père, Pierre Paul Léon, fabricant de chocolat, est mort en 1784. Pauline écrit de lui qu'il était "philosophe» et ne lui a transmis aucun "préjugé ». Sans doute l'aînée de cinq enfants ${ }^{4}$, Pauline a dû aider sa mère, dès l'âge de 16 ans, à continuer son commerce ${ }^{5}$, et à entretenir sa famille.

Dans les sociétés populaires et fraternelles

4 Pauline Léon affirme dans son Précis que c'est très tôt, vers février 1791, qu'elle fut introduite dans plusieurs sociétés; elle cite les Cordeliers, qu'elle fréquentera jusqu'en 
1794, la Société fraternelle des patriotes de l'un et l'autre sexe et celle de Mucius Scaevola.

5 Je ne connais pas de trace d'une participation de Pauline aux séances des Cordeliers, qui furent pour bien des militants une « université populaire ». En 1793, les liens seront étroits entre Cordeliers et Républicaines révolutionnaires.

6 À la Société fraternelle, Pauline côtoie des militants assidus, comme Varlet ou Louise Robert $^{6}$. Nous possédons peu de documents émanant directement de cette Société qui attestent de l'activité de Pauline ${ }^{7}$. Cependant, plusieurs articles du Créole patriote rapportent ses interventions dans les assemblées générales. Par ailleurs, il est intéressant de noter la continuité entre certains thèmes d'intervention de la Société fraternelle et ceux des Citoyennes républicaines. Ainsi, au début février 1791, les sœurs de la Société fraternelle font serment de ne jamais épouser d'aristocrate ${ }^{8}$, tandis que les Républicaines vont promettre en 1793 de peupler la France de petits Marat. La Société fraternelle réclame que les tribunes de l'Assemblée soient réservées au public (janvier 1792) ; ses adhérentes demandent aux députés jacobins de faire supprimer les places réservées9. Les Citoyennes républicaines iront jusqu'à en interdire physiquement l'accès aux porteurs d'invitations.

7 Notons ici que Pauline a signé une Pétition individuelle au corps législatif pour lui demander la punition de tous les conspirateurs qui date de juin 1792 et réclame "une prompte vengeance » contre les ministres monarchistes ${ }^{10}$. La présence sur la première page de signatures, tant masculines que féminines, aux côtés de Pauline, d'une Louise Robert et d'une citoyenne Hardon, toutes deux membres de la Société fraternelle, peut faire penser à une influence de cette société.

En 1793, la Société fraternelle est au premier rang de l'agitation enragée et resserre ses liens avec d'autres sociétés. Dans la même séance du 2 février 1793 où Théophile Leclerc est chargé de rédiger une pétition contre l'argent marchandise, Pauline Léon est accueillie comme étant mandatée par les Défenseurs de la République des 84 départements ${ }^{11}$. C'est, en l'état de notre documentation, la première rencontre de Théophile et Pauline. À partir du 7 février, les deux sociétés tiennent leurs réunions conjointement et agissent souvent de concert. Lors de la séance du dimanche 3 février, Leclerc vient lire son projet rédigé ; Pauline Léon prend la parole après Hébert, pour dénoncer Dumouriez, "notamment sur la persécution qu'il fit éprouver à deux bataillons patriotes accusés par lui injustement ${ }^{12}$ ». Le dimanche suivant 10 février, Pauline fait état d'une dénonciation signée faite à la Commune et à la Société des défenseurs à propos d'un dîner donné chez Garat, ministre de la Justice, auquel auraient notamment assisté Brissot et Beurnonville. Elle « appelle la surveillance de la Société sur ce dernier et demande qu'elle députe deux de ses membres vers celle des Jacobins pour leur communiquer ce fait ${ }^{13}$." Le 17 février, Pauline "fait lecture d'une dénonciation du citoyen Godchaux contre le général Félix Wimpfen ${ }^{14}$ ».

9 Le 9 mars 1792, la Société patriotique de la section du Luxembourg ${ }^{15}$ envoie une délégation à la Société fraternelle pour lui demander l'affiliation ${ }^{16}$. Accédant à cette demande, la Société désigne cinq commissaires pour assister à la prochaine séance du Luxembourg. Parmi eux figurent trois femmes: Pauline Léon, Constance Evrard et Marie-Charlotte Hardon ${ }^{17}$. Pauline participera activement au recrutement de la Société patriotique, présentant personnellement ou appuyant au moins sept candidatures, entre octobre 1792 et septembre $1793^{18}$. 
10 En compagnie de trois autres femmes et de quatre-vingt-huit hommes, Pauline signe la pétition de décembre 1792 par laquelle la Société patriotique réclame la mort du roi. Édité en brochure, le texte contient des menaces à l'égard des députés monarchistes ${ }^{19}$.

11 En février 1793, après plus d'un an d'existence, la Société patriotique se dote d'un règlement qui stipule que le nombre d'adhérentes « ne pourra excéder le cinquième du nombre total de la société »; celles-ci sont éligibles «aux fonctions [...] dans la proportion du cinquième ${ }^{20} »$. De plus, les hommes sont admis à 16 ans et les femmes à 21 ans (ou 14 ans si leurs parents sont adhérents). Ces restrictions visant les femmes, notamment les plus jeunes, ont pu contribuer à faire mûrir l'idée d'une société exclusivement féminine.

La citoyenneté des femmes et l'exercice de la violence physique

12 Pauline fait état de plusieurs incidents violents dont elle est, en compagnie de sa mère ou d'amies proches, la victime et/ou l'acteur. En février 1791, elle défenestre un buste de La Fayette "chez Fréron ${ }^{21}$ ". Elle est sérieusement molestée par les partisans du même La Fayette en juin 1791, puis fait le coup de poing pour défendre une amie contre la famille d'un garde national, le 17 juillet 1791, au retour du Champ-de-Mars. Ce dernier incident est attesté dans le procès-verbal de l'interrogatoire par le commissaire de police de la section Fontaine-de-Grenelle de son amie Constance Evrard ${ }^{22}$.

Des femmes révolutionnaires participent, armées, à toutes les journées insurrectionnelles. Pauline rapporte un autre incident significatif : " Au 10 août 1792, après avoir passé une partie de la nuit à la section de la Fontaine-de-Grenelle, je me mis le lendemain, armée d'une pique, dans les rangs des citoyens de cette section pour aller combattre le tyran et ses satellites. Ce ne fut qu'à la prière de presque tous les patriotes que je consentis à me défaire de mon arme en faveur d'un sans-culotte; je ne la lui remis cependant qu'en l'incitant à s'en bien servir.» Durant l'insurrection antigirondine de mai-juin 1793, plusieurs Républicaines sont arrêtées. Pauline signe, comme présidente, l'arrêté par lequel les Citoyennes républicaines révolutionnaires répondent de Marie Anne Vilquin, détenue pour avoir menacé trois hommes de son couteau ${ }^{23}$

14 Parmi les pétitions, " en grand nombre » assure-t-elle, au bas desquelles on trouve la signature de Pauline Léon, la plus connue est l'Adresse individuelle à l'Assemblée nationale par des citoyennes de la capitale. Elle présente le double intérêt d'être probablement de la main même de Pauline et de synthétiser le dilemme des femmes révolutionnaires : se présenter comme des «citoyennes » qu'elles ne sont pas, en réclamant le droit de porter des armes à ceux qui s'y opposent.

15 L'adresse a dû être rédigée à la fin février $1792^{24}$; elle est d'abord lue devant la Société fraternelle séante aux Minimes qui, sous la présidence du jacobin Tallien, en ordonne à l'unanimité l'impression et la distribution. Au bas de la version manuscrite conservée aux Archives nationales, la première signature, et la seule figurant dans la version imprimée sur ordre de l'Assemblée, est libellée « fille Léon ${ }^{25}$ ». Environ trois cent dix noms suivent celui de Pauline, dont celui de sa mère. Les mentions «femme ", " fille » et « veuve » permettent de repérer de nom-breux couples mère-fille.

"Législateurs, commence le texte, dont je souligne certains termes, des femmes patriotes se présentent devant vous pour réclamer le droit qu'a tout individu de pourvoir à la défense de sa vie et de sa liberté. " L'oratrice s'adresse d'abord, c'est la seule fois, aux députés en tant que législateurs et non en tant qu'hommes. "Nous sommes citoyennes ", 
c'est ce parti pris qui ouvre le troisième alinéa. «Vos prédécesseurs ont remis le dépôt de la Constitution dans nos mains, aussi bien que dans les vôtres: eh! comment conserver ce dépôt, si nous n'avons des armes pour le défendre [...]. » Réitérée plus loin dans le texte, la tentative de présenter la citoyenneté des femmes comme un fait acquis annonce la stratégie des Citoyennes républicaines révolutionnaires et des sociétés populaires qui affirmeront une légitimité politique dans l'approbation ostentatoire de la Constitution de 1793, palliatif de l'absence du droit de vote, que les Citoyennes républicaines ne réclameront d'ailleurs pas ${ }^{26}$.

17 «Oui, Messieurs, ce sont des armes qu'il nous faut; et nous venons vous demander la permission de nous en procurer. » C'est dorénavant aux hommes (6 occurrences pour Messieurs) et non plus aux législateurs ou à des citoyens que s'adresse le texte. Le changement de ton n'est pas sans pertinence - les «citoyennes " savent qu'elles sont d'abord regardées comme femmes par des hommes -, mais il marque et entrâne un recul par rapport à la stratégie du fait accompli. «Ne croyez pas cependant, ajoutentelles, que notre dessein soit d'abandonner les soins, toujours chers à nos cœurs, de notre famille et de notre maison [...].» Du fait accompli, on retourne au statu quo: insuffisant à rassurer les hommes, ce langage confirme l'assujettissement des femmes.

18 "Vous ne pouvez nous refuser, martèle Pauline, et la société ne peut nous ôter ce droit que la nature nous donne; à moins que l'on ne prétende que la Déclaration des droits n'a point d'application pour les femmes, et qu'elles doivent se laisser égorger comme des agneaux [...]». Des "hommes froids» pourraient bien qualifier le zèle des citoyennes « de fanatisme et d'exagération » - ils ne s'en privent pas, en effet -, mais ce n'est que le « résultat naturel d'un cœur brûlant de l'amour du bien public ». Après leur cœur brûlant, c'est leur corps sexué que les pétitionnaires évoquent : « N’y aurait-il pas de la cruauté à nous condamner d'attendre, dans nos maisons, une mort honteuse, et toutes les horreurs qui la précéderaient.» On peut douter de l'efficacité, pour convaincre des hommes, de l'insistance sur le statut de proie érotique des femmes, promises - par leur faiblesse et leurs attraits physiques - au viol et à la honte. Avant d'en arriver aux modalités pratiques de l'armement (fourniture, exercice, encadrement), le texte revient sur l'idée d'une citoyenneté acquise, mais c'est pour reconnaître qu'elle n'échoit aux femmes que par l'intermédiaire des hommes, législateurs et/ou époux : « Si [...] vous vous refusiez à nos justes demandes, [...] des femmes que vous avez élevées au rang de citoyennes en rendant ce titre à leur époux, des femmes qui ont goûté les prémices de la liberté [...] mourront plutôt. »

19 Annie Geffroy a souligné, à propos de la querelle vestimentaire et symbolique qui fournira l'occasion de disperser les Citoyennes républicaines révolutionnaires, que « la femme en bonnet rouge est, dans l'imaginaire masculin, dotée de pantalons et d'armes, menaçante, phallique ${ }^{27} »$. Ajoutons que cet imaginaire masculin et machiste est alimenté par une littérature et un folklore érotique où la "pique ", arme naturelle et emblématique du peuple insurgé dès l'été $1789^{28}$, est aussi - depuis le XVI ${ }^{\mathrm{e}}$ siècle - une métaphore du phallus, que l'on retrouve chez Piron et chez Sade ${ }^{29}$.

20 En mai 1793, les Citoyennes républicaines annoncent qu'elles veulent former des " compagnies d'amazones", par la réquisition des femmes de 18 à 50 ans $^{30}$. Le 27 mai, une députation de la Société, dont nous savons qu'à cette date Pauline est présidente, se présente aux Jacobins. Son discours associe sans détours protestation féministe et mot d'ordre insurrectionnel : «Il est temps que vous ne voyiez plus en nous des femmes serviles, des animaux domestiques ${ }^{31} »$. Recul tactique ou conséquence de dissensions 
internes : début juillet 1793, le premier des vingt-sept articles de leur règlement, le moins clairement rédigé de tous, stipule que, si le but de la Société est bien de faire s'armer les citoyennes, elles sont «néanmoins libres» de ne pas le faire. Pour autant, ces militantes ne renoncent pas à s'armer: lorsque l'on perquisitionne chez Claire Lacombe en octobre 1793, on trouve vingt piques ${ }^{32}$.

La Société des Citoyennes républicaines révolutionnaires

Le 10 mai 1793, des citoyennes déclarent au secrétariat de la municipalité leur intention de « former une société où les femmes seules pourront être admises. [...] Elle portera le nom de Société Républicaine révolutionnaire et se réunira à la bibliothèque des Jacobins, rue Saint-Honoré33. " La Société se présente aux Jacobins dès le 12 ; elle y revient le 19, et l'on peut présumer la présence de Pauline Léon. En effet, c'est une députation mixte avec les Cordeliers qui vient réclamer, dans un projet d'adresse à la Convention ${ }^{34}$, l'arrestation des Girondins et des suspects, la formation de tribunaux révolutionnaires dans les départements et les sections, que l'armée révolutionnaire de Paris soit portée à 40000 hommes, que l'on distribue des terres aux soldats et qu'un impôt sur les riches rembourse "aux hommes de peine» ce qu'ils dépensent quotidiennement de pain au-dessus de 3 sols la livre.

Jacques Roux rendra hommage au rôle des « citoyennes révolutionnaires [...] apôtres fidèles de la révolution [à qui] nous devons, en partie, la gloire d'avoir sauvé la République dans les journées des 31 mai et 2 juin derniers ${ }^{35}$. » Pauline et ses camarades éprouvent cependant des difficultés à se faire admettre, aux côtés des insurgés d'abord, à la Convention ensuite. Le 2 juin, Pauline conduit une délégation de Citoyennes républicaines révolutionaires qui souhaitent être admises à la barre ${ }^{36}$, demande vite oubliée dans le tumulte et les débats qui aboutissent à l'assignation à résidence de vingt-deux Girondins.

En juillet 1793 est publié le Règlement de la Société des Citoyennes républicaines révolutionnaires de Paris ${ }^{37}$ (daté du 9). Dans le préambule, les militantes jurent de ne pas donner prise aux reproches que les hommes adressent volontiers aux femmes: "Convaincues que sans mœurs et sans principes il n'y a pas de liberté, et que pour bien remplir ses devoirs domestiques il faut connaitre ses devoirs sociaux [...] elles veulent bannir toute personnalité, jalousie, rivalité, envie, et justifier leur titre.» Le programme peut paraître anodin : instruction mutuelle, étude de la Constitution et des lois de la République, défense de tout individu victime de l'arbitraire; il s'agit enfin de "s'occuper des affaires publiques». C'est aller beaucoup moins loin que les Républicaines ne l'ont fait en mai devant les Jacobins. Plus question d'exterminer les scélérats, ni de former des légions d'amazones. Le document est signé par la présidente Rousaud et par quatre secrétaires: Potheau, Monier, Dubreuil, et Pauline Léon. On remarque l'absence de la signature de Claire Lacombe, qui ne semble s'être affirmée que progressivement comme animatrice de la Sociétée ${ }^{38}$. Le 10 juillet, lendemain de la date de rédaction du Règlement, Pauline vient chercher l'appui des Jacobins pour une adresse qu'elle se propose de présenter le lendemain à la Convention et qui réclame l'exclusion des nobles de tous les emplois, notamment d'officiers ${ }^{39}$.

Le 13 juillet 1793, Marat est assassiné. Pendant un mois, l'activité des républicaines va se concentrer presque exclusivement sur le culte du martyr. E. Charavay a signalé une «Délibération de la Société des Républicaines révolutionnaires [...], relative à l'érection d'un obélisque à la mémoire de Marat, sur la place du Carrousel », datée du 20 juillet, et signée de Pauline Léon ${ }^{40}$. Le 15 août, elle dirige une députation des Citoyennes 
révolutionnaires qui viennent solliciter l'affiliation et la correspondance "avec leurs frères Jacobins ", ce qui leur est accordé. « Elles demandent ensuite que la Société [des Jacobins] nomme des commissaires pour examiner l'état des dépenses de l'obélisque élevé à Marat. " Les Républicaines, qui ont tenu un rôle de premier plan dans les cérémonies d'inhumation (17 juillet), puis de translation du cœur de Marat dans le local des Cordeliers (28 juillet), inaugurent enfin leur obélisque le 18 août ${ }^{41}$. Le soir même, après la fête, Claire Lacombe conduit aux Jacobins une nouvelle députation mixte des commissaires des sections et des Citoyennes républicaines. Elle annonce que ces dernières "vont veiller au salut public ${ }^{42}$ ». Les Républicaines peuvent se consacrer à d'autres tâches que l'édification du symbole Marat.

Nous ne disposons plus pour la fin 1793 que d'un document qui soit certainement de la main de Pauline. Il s'agit d'une dénonciation contre l'épicier Le Doux, rue du Sépulcre, pour « mauvais propos ${ }^{43}$ ", lesquels consistent surtout à se plaindre des pillages, que les Citoyennes révolutionnaires condamnent d'ailleurs ${ }^{44}$. Pourtant, l'unanimité ne devait pas être aussi parfaite que voulait bien le dire leur état-major puisqu'elles annoncent à la Commune quatre mois plus tard, le 24 octobre, qu'elles ont « chassé de leur Société des citoyennes convaincues d'avoir reçu de l'argent, et suscité des émeutes à l'occasion des craintes occasionnées pour le savon ${ }^{45}$. " Soucieuses de donner des gages aux autorités, les Citoyennes révolutionnaires font prendre corps rétrospectivement aux calomnies de leurs adversaires, et ce à quelques jours de la dissolution de leur société.

«Femme Leclerc»

Pauline Léon épouse Théophile Leclerc en novembre $1793^{46}$ et déclare reprendre à cette époque le commerce familial de chocolat.

Certainement inquiète de la répression qui frappe les Hébertistes et les Cordeliers ${ }^{47}$, Pauline rejoint Leclerc le 17 mars 1794, à La Fère (Aisne), où il est mobilisé. C'est là qu'ils sont arrêtés le 3 avril, sur ordre du Comité de Sûreté générale. Ramenés à Paris, ils sont incarcérés au Luxembourg le 6 avril.

Après le 9 Thermidor, Pauline cherche des appuis. Le 18 thermidor (5 août 1794), elle écrit au "sensible Tallien » (qu'elle a connu en 1792), plaide la cause des « huit cent personnes détenues » et de Leclerc, privé de promenade, de papier et d'encre ${ }^{48}$. Le 19 thermidor ( 6 août), elle s'adresse aux «représentants » pour réclamer au moins le prompt examen de leur cas. Elle confirme que Leclerc et Réal sont au secret pour avoir « recueilli des faits contre les complices du tyran Robespierre qui devaient [les] faire égorger ${ }^{49}$ ». Le lendemain, Réal et Leclerc sont amenés devant le Comité de Sûreté générale. Réal est libéré; Pauline et Théophile le seront le 22 août.

Suivant à la lettre un passage édifiant du Précis - « Je me livrais tout entière au soin de mon ménage et je donnais l'exemple de l'amour conjugal et des vertus domestiques qui sont à la base de l'amour de la patrie »-, Dominique Godineau date de 1794 la dernière trace connue de Pauline. Or, le dossier de police de François Léon, frère de Pauline, dont les principales pièces ont été publiées par M. D. Sibalis en 1982, nous apprend beaucoup sur Pauline et sa famille en $1804^{50}$. Arrêté et détenu trois mois et demi pour avoir, avec un nommé Sornet, rédigé et collé des papillons hostiles à Bonaparte, François revendique crânement ses opinions : «Je suis très républicain ; [...] on m'a dit que le premier Consul allait être Empereur, [...] cela ne me convient point [...]. On se dit libre et personne ne l'est; on veut faire prendre des livrets aux ouvriers. » Sornet le décrit comme " un jacobin prononcé ainsi que toute sa famille ». Précisément, le même dossier contient une lettre, du 22 juillet 1804, que Pauline adresse à Réal, co-détenu de 
Leclerc en 1794, devenu l'un des responsables de la Police générale. La lettre, par laquelle Pauline sollicite l'élargissement de son frère, nous apprend qu'elle exerce à Paris la profession d'institutrice. Signée «femme Leclerc », elle indique que Théophile est vivant en 1804.

Libéré, François Léon doit s'éloigner de Paris, et des côtes. Désormais fiché comme " anarchiste prononcé, difficile à corriger ${ }^{51}$ ", la police suit sa trace entre Dijon et Lyon (1805) ; en 1807, il est à Châteauroux ; en juin il obtient un passeport pour Limoges.

31 Le 17 janvier de cette même année 1807, sa sœur Antoinette Léon épouse Pierre Antoine Louis Allut, imprimeur, rue de l'École de Médecine, à Paris ${ }^{52}$. Un rapport de police, non daté mais antérieur à 1812, nous apprend que Allut est un ancien tailleur de pierre, imprimeur depuis la Révolution et qu'il a établi chez lui, sans succès, un cabinet de lecture. Le même document décrit « un pauvre être [sic]», borgne, et qui «ne fait pas grand chose $^{53} »$. Son bail expire le 1er janvier 1812. Le baron de Pommereul, directeur général de la Librairie, lui propose d'aller à Napoléon-Vendée, où il s'installe en juillet 1812 et devient imprimeur de la Préfecture ${ }^{54}$, malgré des rapports de police dénonçant ses mœurs, ses opinions contraires au gouvernement, et ses fréquentations ${ }^{55}$. Une note au ministre de la Police générale (14 mai 1817) recommande la surveillance des imprimeurs Allut et Ferré, «à raison des opinions qu'ils avaient manifestées [...], surtout pendant les trois mois de l'usurpateur et des liaisons qu'ils ont entretenues et entretiennent journellement avec des personnes ennemies du gouvernement. » Allut aurait donc, notamment pendant les Cent-Jours, exprimé des sympathies bonapartistes ou républicaines. Les rapports de police ne sont hélas pas plus précis sur les «personnes ennemies du gouvernement" qu'Allut fréquente quotidiennement. Malgré la cohérence dans le temps de ces renseignements défavorables, l'imprimeur ne semble pas avoir encouru d'autre sanction qu'une peine d'un mois d'emprisonnement et 200 francs d'amende, en mars 1823, pour avoir édité sans autorisation la Feuille judiciaire, commerciale et littéraire du département de la Vendée ${ }^{56}$.

Nous ignorons la date précise de l'installation de Pauline Léon auprès de sa sœur, sinon qu'elle se situe entre 1812, date d'arrivée des époux Allut à Napoléon-Vendée, et 1835. À ce jour, la seule attestation connue de son domicile vendéen, avant son propre décès, figure sur un registre de décès où sont portés les légataires de son frère Antoine Paul Louis, décédé le 31 mars $1835^{57}$.

33 Allut meurt le 21 janvier 1832 ; Marie Reine Antoinette sollicite, en février de la même année, de reprendre à son nom le brevet d'imprimeur-libraire de son mari, ce qui lui est accordé58 ${ }^{5}$. Le 7 janvier 1840, Antoinette se désiste en faveur de son frère François ${ }^{59}$. Entre-temps, Pauline Léon meurt à Bourbon-Vendée, le 5 octobre 1838, à l'âge de soixante-dix ans, rentière, dans sa maison de la rue de Bordeaux. L'imprimeur Leconte, indiqué comme étant le neveu de la défunte (il a épousé la nièce de Pauline, Joséphine Reine Pauline ${ }^{60}$ ) et Pierre Auguste Richard, imprimeur, en font la déclaration à l'adjoint au maire de Bourbon-Vendée ${ }^{61}$.

35 Ainsi disparaît, dans un noyau familial sœurs/frère dont le temps n'a altéré ni les solidarités ni les opinions, une militante exemplaire de la Révolution française. C'est dans son bouillonnement qu'elle se découvre révolutionnaire, puis républicaine. C'est dans la logique contrariée de la participation des femmes qu'elle contribuera à créer un outil de lutte autonome de femmes, dont l'appellation réunit les termes «citoyennes, républicaines et révolutionnaires». Malgré les limites théoriques de ce féminisme pratiqué, il incarne en 1793 la radicalité du courant Enragé, dont il est la composante la 
plus originale. Ainsi, avant d'être interdite avec tous les clubs de femmes le 30 octobre 1793, la société dont Pauline est cofondatrice est fermée manu militari au cri de "Vive la République ! À bas les [femmes] révolutionnaires ${ }^{62}$ ! ». L'offensive contre le mouvement sans-culotte, qui conduira à l'interdiction des sociétés populaires, commence par la fermeture du club des femmes, et l'élision du mot même.

\section{NOTES}

1.Daniel GUÉRIN considère les Citoyennes républicaines révolutionnaires « en quelque sorte [comme] la section féminine du mouvement des Enragés ", La lutte de classes sous la Première République. Bourgeois et «bras nus " 1793-1797, Paris, Gallimard, 1946, t. I, p. 244. Albert SoBoul écrit qu'en août 1793, « les Jacobins ignoraient encore que, derrière les citoyennes Léon et Lacombe, Leclerc agissait », Mouvement populaire et gouvernement révolutionnaire en l'an II, Paris, Flammarion, 1973, p. 99.

2.Robert Barrie Rose, The Enragés, Socialists of the French Revolution? Melbourne University Press, 1965 ; Marie CERATI, Le Club des citoyennes républicaines révolutionnaires, Paris, éd. sociales, 1966 ; Dominique GodineAu, Citoyennes tricoteuses, Alinéa, 1988 (reprint, Perrin, 2004) ; Claude GuilLon, Deux Enragés de la Révolution, Leclerc de Lyon et Pauline Léon, La Digitale, 1993 (figurent dans l'ouvrage l'ensemble des textes rédigés ou signés par les deux Enragés, y compris les vingt-quatre numéros de L'Ami du Peuple). 3.Dans un texte rédigé ou plus probablement dicté par elle le 4 juillet 1794, à la maison d'arrêt du Luxembourg, adressé au Comité de Sûreté générale : Précis de la conduite révolutionnaire de dame Pauline Léon, femme Leclerc (Archives Nationales : F7 4774/9). Sauf indication contraire, les citations de Pauline sont tirées du Précis.

4.L'âge de l'un[e] des enfants nous demeure inconnu. Nous connaissons deux frères et une sœur : Antoine Paul Louis, dont nous savons seulement qu'il est né en 1772 et qu'il meurt le 31 mars 1835, ouvrier, demeurant 219, faubourg Saint-Martin ; Marie Reine Antoinette, née le 17 janvier 1778 ; François Paul Mathurin, né le 23 octobre 1779. 5.Au 356 de la rue de Grenelle, qui constituait la limite sud de la section Fontaine-deGrenelle et la limite nord de la section de la Croix-Rouge, qui prit en 1793 le nom de Bonnet-Rouge ou de la Liberté. Il semble que le domicile des Léon ait été dans le ressort de la première, mais elle indique la section du Bonnet-Rouge lors de son mariage en 1793 et de son arrestation en 1794.

6.Voir Annie GeFFroy « Louise de Kéralio, traductrice, éditrice, historienne et journaliste, avant 1789 ", in Lectrices d'Ancien Régime, dir. Isabelle BROUARD-ARENDS, Presses Universitaires de Rennes, 2003 et « Louise de Kéralio-Robert, pionnière du républicanisme sexiste ", dans ce volume.

7.Un lapsus calami m'avait fait intervertir (Deux Enragés de la Révolution, p. 100) Société fraternelle et Société patriotique de la section du Luxembourg, et attribuer à la première les bulletins d'affiliation qui concernent la seconde. Voir plus loin la rectification de cette erreur.

8.L'Orateur du peuple, $\mathrm{n}^{\circ} 48$, t. IV ; Révolutions de Paris, $\mathrm{n}^{\circ} 83,5$ au 12 février. 
9.Rapport du 18-19 mai 1793 ; Alexandre TUETEY, t. IX, nº 606.

10.AN : C $1522, \mathrm{n}^{\circ} 2705$ p. 2.

11.Le Créole patriote, $\mathrm{n}^{\circ} 271,4$ février 1793.

12.Le Créole patriote, $\mathrm{n}^{\circ} 273,5$ février 1793.

13. Le Créole patriote, $\mathrm{n}^{\circ} 289,13$ février 1793. C'est probablement Pauline qui a déjà présenté la dénonciation aux Jacobins, ce même dimanche 10 février ; La Société des Jacobins, François-Alphonse AULARD, t. V, p. 23-24.

14.Le Créole patriote, $\mathrm{n}^{\circ}$ 305. Le baron Louis-Félix de Wimpfen (1745-1814), commandant l'armée des côtes de Cherbourg, rejoindra en juin les insurgés fédéralistes.

15.Société et section prendront en octobre 1793 le nom de Mucius Scaevola. Voir R.B. ROSE, « Nursery of sans-culottes : The Société patriotique of the Luxembourg Section, 1792-1795 », Bulletin of the John Rylands Library, Manchester, vol. 64, automne 1981, $\mathrm{n}^{\circ} 1$, p. 218-245, et Raymonde MONNIER, L'espace public démocratique. Essai sur l'opinion à Paris de la Révolution au Directoire, Paris, Kimé, 1994.

16.BNF : Ms. NAF 2705, f. 164.

17. Constance Evrard est une intime de Pauline, Marie-Charlotte Hardon, une ouvrière en linge de vingt-quatre ans ; elles suivront bientôt Pauline Léon chez les Citoyennes révolutionnaires, Archives de la Préfecture de Police (APP) : AA 158, f. 476.

18.BNF : ms. NAF 2705, f. 216, 222, 223, 235, 239, 264, 265, 266. Candidatures de :

Bourgeois, Marie Catherine Félicité (s.d.) ; Chevalier François Guillaume (octobre 1792) ; Lelièvre Denise, 337 rue du Four (octobre 1792) ; Boufaud (orth. incertaine), JeanBaptiste, homme de loi, rue des Vieux-Augustins (s. d.) ; Husard, Marie et CharlesGuillaume, 257 rue du Bac (20 septembre 1793), Laugier, Balthazar-Marie, 1107 rue de Grenelle (il est donc voisin de Pauline ; 20 septembre 1793). Jacobin, Laugier a été avec Leclerc membre du Comité central révolutionnaire du 31 mai. Marie Berdin, épouse Husard, domiciliée dans le ressort de la Fontaine-de-Grenelle, sera arrêtée le 28 janvier 1795 pour avoir fréquenté les Jacobins et organisé des réunions chez elle. Cf. Répertoire du personnel sectionnaire parisien en l'an II, Albert SOBOUL et Raymonde MONNIER, Publications de la Sorbonne, 1985, p. 433 et 438.

19.Adresse au peuple par la Société patriotique de la section du Luxembourg, 6 p., BHVP : $600819\left(n^{\circ} 4\right)$.

20.BNF : ms NAF 2705, f. 6.

21.Alexandre TUETEY, t. I, n² 2517. L'épisode peut paraître obscur dans la mesure où Louis Stanislas Fréron, Cordelier et rédacteur de L'Orateur du peuple ne ménageait pas ses critiques à La Fayette. Il est probable que l'expédition visait plutôt Anne Françoise Fréron, mère du publiciste ou son oncle l'abbé Royou, directeur de L'Ami du Roi. 22.APP : AA 148, f. 30. C'est la première trace dans les archives de l'activité militante de Pauline.

23.APP : AA 90, f. 562, 563.

24.La plus souvent consultée des deux versions imprimées indique la date fautive du 6 mars 1791, qui a abusé chercheurs et éditeurs au lieu de 1792. On retrouve l'erreur dans Cahiers de doléances des femmes et autres textes (Des Femmes, 1981), et Les Femmes dans la Révolution française (EDHIS, 1982). Rectifiée dans le même volume par Dominique Godineau, elle figure dans le texte de M. Bernd JESCHONNEK, « Pauline Léon et la société des citoyennes républicaines révolutionnaires ", colloque international de Toulouse, Les Femmes et la Révolution française (vol. I, 1989). 
25.BNF : $8^{\circ}$ Lc33 3 x ; AN : Microfilm C 145, CI 190. Sous la même cote : un message de la main de Pauline adressé au président de l'Assemblée. Fort obséquieux, il se clôt néanmoins sur la formule : « Nous avons l'honneur d'être [...] vos concitoyennes. » 26.Pauline écrit : « C'est dans le sein de la Convention nationale que j'exprimai au nom des citoyennes de ma section leur joie et leur satisfaction sur l'achèvement de la constitution. »C'est sans doute le 24 juin 1793, date à laquelle les Citoyennes républicaines révolutionnaires doivent se mêler au cortège des sociétés populaires et des sections ; Journal de la Montagne, 23 juin 1793.

27.Annie GEFFROY, «A bas le bonnet rouge des femmes !" (octobre-novembre 1793) », Les femmes et la Révolution française, Toulouse, Presses Universitaires du Mirail, 1990, p. 345-351.

28. Élisabeth LIRIS, article « Piques » du Dictionnaire historique de la Révolution française, Paris, PUF, dir. Albert SoBoul, 1989.

29.Sade décrit dans Justine deux valets « débarrassés de leurs culottes, [qui] s'avancent la pique à la main ». Cf. la note de Michel DeLon dans le t. II des Euvres, Pléiade Gallimard, 1995, p. 1257 et Pierre GUIRAUD, Dictionnaire érotique, Payot, 1993.

30.Aux Jacobins, le 12 mai 1793 ; Journal des débats, $\mathrm{n}^{\circ} 412$; La Révolution de 92, nº 238. Devant l'assemblée générale de la section du Muséum, le lendemain; Bibliothèque Victor Cousin, Ms. 117, f. 147-148.

31.Philippe-Joseph-Benjamin BuCHEZ et Pierre-Célestin ROUX-LAVERGNE, Histoire parlementaire de la Révolution française [...], Paris, Paulin, 1834-1838, t. 27, p. 275-276. Je souligne.

32.AN : T 10012.

33.Moniteur, t. XVI, p. 362. Cette source ne mentionne pas le terme citoyennes. 34.Si l'on en croit la Chronique de Paris du 22 mai, qui publie le même texte, lu le lendemain 20 mai à la Commune, l'initiative revient aux Citoyennes républicaines révolutionnaires puisque le texte porte la mention : «La société des Cordeliers adhère à l'adresse, et en a arrêté l'impression. » Gustave-Alphonse AULARD, op. cit., t. 5, p. 198-199; AN : C 355, nº 1865.

35.Devant la Commune, le 21 juin ; Journal de la Montagne, $\mathrm{n}^{\circ} 22,23$ juin 93.

36.AN : C 258, $\mathrm{n}^{\circ}$ 528. Adressée à Durand-Maillane, la demande est de la main de Pauline Léon.

37.Cf. le texte complet (Bibliothèque Historique de la Ville de Paris : 958 939) dans Claude GuILlon, Deux Enragés de la Révolution, op. cit.

38.Sa première apparition attestée comme députée de la société date du 26 juin 1793 , aux Jacobins (Gustave-Alphonse AULARD, op. cit., t. V.). Contrairement à ce qu'a écrit Robert Barrie Rose, The Enragés [...], op. cit., p. 75, il n'y a aucun doute sur la présence simultanée de Claire et Pauline au sein de la Société.

39.Journal des débats, 14 juillet 1793.

40.Inventaire des autographes et des documents historiques composant la collection de $M$. Benjamin Fillon, par étienne CHARAVAY, Paris, 1877, p. 75, n 556 (17). Pauline fait certainement office de secrétaire, la présidence de la citoyenne Colinger étant attestée les 17 et 27 juillet. En application de l'art. XI du règlement, le document devait porter les signatures de la présidente et de deux secrétaires. Journal de la Montagne, $\mathrm{n}^{\circ}$ 57, 28 juillet 1793. Le texte est lu aux Jacobins le 26.

41. Annales de la République française, $\mathrm{n}^{\circ} 234,21$ août 93.

42.Journal des débats, 20 août 93. 
43. $\mathrm{AN}$ : $\mathrm{BB} 372, \mathrm{n}^{\circ} 69$. Nous ignorons si la dénonciation a eu des suites.

44. Affiches de la Commune, $\mathrm{n}^{\circ} 13,28$ juin 93.

45. Affiches de la Commune, $\mathrm{n}^{\circ} 123,25$ octobre 93.

46.Le contrat de mariage, rédigé par Me Ballet, porte la date du 22 brumaire $[12$ novembre 1793] ; il est enregistré le 8 frimaire an II. Les biens du futur époux consistent en la somme de trois cents livres en effets et linge, ceux de la future en la somme de mille livres tant en argent qu'en effets et en deux actions sur la caisse de bienfaisance dite Lafarge. Pauline est donc plus riche que son époux et le contrat, qui met 150 livres en commun, et prévoit un douaire de 400 l. et un préciput de $300 \mathrm{l}$. aurait été favorable à Leclerc s'il n'était mort avant sa femme. AN : ET/LXV/521, f. 31.

47.I est possible qu'elle ait assisté à la séance du 4 mars 1794 où une délégation de la Société populaire de Mucius Scævola vient appuyer l'idée d'insurrection. Cf. la dénonciation de Marie Jeanne Élisabeth Brocart-Jolly, 16 mars 1794, et la déposition de Farjaire, Alexandre TUETEY, Répertoire général des sources manuscrites de l'histoire de Paris pendant la Révolution française, Paris, Imprimerie nouvelle, 1890-1914, t. XI, nº 36 et 43. 48.Je remercie Serge ABERDAM qui m'a signalé l'existence de cette lettre, prélevée par le conventionnel Laloy dans les papiers du Comité de Sûreté générale et M. François Petrazoller, directeur des Archives départementales de Haute-Marne qui m'en a transmis copie (ADHM F18).

49.AN : F7 47749.

50.M. D. SIBALIS, « Un sans-culotte parisien en l'an XII, François Léon, frère de Pauline Léon ", AHRF, n² 248, avril-juin 1982, p. 294-298 ; Claude GuILLoN, Deux Enragés..., op. cit., 1993 , p. 85 et suiv.

51.AN : F7* $^{*} 702$.

52.AN : F18 509.

53.AN : F18 2371, dossier Allut. Une expertise évalue ses quatre presses à 897 francs ; son loyer annuel est de 330 francs et son imposition en 1810 de 124,95 francs.

54.AN : F18 509, certificat de résidence du maire de Napoléon-Vendée.

55.Cf. pour 1815 : AN : F18 509 et AD Vendée 4 M 389 ; pour 1823 : AN : F18 2105. Le Préfet de Vendée demande la suppression de son brevet (s.d., après 1814), AD Vendée 4 M 389.

56.AN : F18 2105.

57.« Anne Pauline Léon, veuve Leclerc, Bourbon-Vendée »; AP DQ8 703 f. 10. Nous apprenons du même coup qu'à cette date, Leclerc ne vit plus.

58. Brevet d'imprimeur $n^{\circ} 2743$ et brevet de libraire ${ }^{\circ} 3455$, accordés le 28 février 1832. AN : F18 2105, dossier Allut veuve, Marie Reine Antoinette Léon.

59.AN : F18 2105, Léon François. Le certificat d'aptitude fourni par François à l'appui de sa demande est signé par trois personnes, dont sa sœur Antoinette, et Camille Leconte, imprimeur qui a épousé une fille d'Antoinette.

60.L'acte de naissance de cette nièce, à laquelle on a donné le prénom de sa tante, pourrait attester de la présence de celle-ci à Bourbon-Vendée, si comme il est plausible elle en était la marraine. Je n'ai pas eu le temps de suivre cette piste. Antoinette signale l'existence de ses deux filles, et le mariage de l'ânée avec Leconte dans sa demande de brevet (AN : F18 2105). Le prénom de l'ânée est indiqué dans Mémoire écrite de La Rochesur-Yon. Dictionnaire des écrivains, éditeurs, imprimeurs, journaux et journalistes 1804-2004, La Roche-sur-Yon, éd. Siloe, 2004 ; je remercie M. Frustier, son directeur, de me l'avoir signalé. Hélas, ce dictionnaire ne connaît Antoinette que comme épouse Allut, et non comme imprimeur breveté ; il ignore François Léon. 
61.AD Vendée, État-civil, Naissances Mariages Décès 1838, f. 343, acte $n^{\circ} 215$. Je remercie M. Feuermann de m'avoir signalé l'existence de cet acte.

62. Les Révolutions de Paris, $\mathrm{n}^{\circ} 215,13-20$ novembre 1793, cité dans Dominique GoDINEAU, op. cit., p. 171.

\section{RÉSUMÉS}

Fille d'un artisan chocolatier, âgée de 21 ans en 1789, Anne Pauline Léon participe, dès la prise de la Bastille, aux journées révolutionnaires et à l'activité des sociétés populaires. Elle est pétitionnaire pour l'armement des femmes puis cofondatrice de la Société des citoyennes républicaines révolutionnaires (juillet 1793), outil de la lutte autonome des femmes révolutionnaires, qui contribue à la rapprocher du courant des Enragés, dont elle épouse le plus jeune animateur, Théophile Leclerc. Institutrice à Paris, en 1804, elle réclame la libération de son frère, détenu pour des écrits hostiles à Bonaparte. Elle rejoindra à Bourbon-Vendée (La Rochesur-Yon), sa sœur et son frère qui exercent la profession d'imprimeur. Elle y décède le 5 octobre 1838, à l'âge de 70 ans.

Pauline Léon, a Revolutionary Republican. The daughter of a artisanal chocolate maker, Anne Pauline Leon, aged 21 in 1789, participated from the fall of the Bastille in the different revolutionary journees, and was active in the societes populaires. Petitionner for the arming of women, then co-founder of the Societe des citoyennes republicaines revolutionaires (July 1793), a tool in the autonomous struggle of revolutionary women, she contributed to bringing them closer to the thinking of the Enrages, the youngest of whom she married, Theophile Leclerc. School teacher in Paris in 1804, she demanded the release of her brother, imprisoned for his hostile writing of Bonaparte. She joined in Bourbon - Vendée (La Roche-sur-Yon), her sister and her brother who exercised the profession of printer. She died on October 5, 1838, aged 70.

\section{INDEX}

Mots-clés : Citoyennes républicaines révolutionnaires, Société fraternelle des patriotes des deux sexes, enragés, Marat, sans-culottes, piques

\section{AUTEUR}

CLAUDE GUILLON

claude.guillon@internetdown.org 